\title{
Artificial Fish Swarm Algorithm Based Optimal Sensor Placement
}

\author{
Zhen-rui Peng ${ }^{1}$, Yu Zhao ${ }^{2}$, Hong Yin ${ }^{1}$ and An Pan $^{1}$ \\ 1. School of Mechatronics Engineering, Lanzhou Jiaotong University, China \\ 2. School of Electronic Information and Electrical Engineering, Tianshui Normal \\ University, China \\ pengzr@mail.lzjtu.cn
}

\begin{abstract}
In order to gain more information reflecting bridge health status, with fewer sensors, a method based on artificial fish swarm algorithm (AFSA) is proposed to solve optimal sensor placement $(\mathrm{OSP})$ problem. The algorithm takes modal assurance criterion (MAC) matrix obtained from modal analysis of an arch bridge structure as the objective function. Four typical behaviors of artificial fish are applied to search the optimal solution. The results show that AFSA is more effective than the particle swarm optimization (PSO) method, in achieving optimal sensor placement.
\end{abstract}

Index terms: optimal sensor placement, artificial fish swarm algorithm, modal analysis, finite element model

\section{Introduction}

Large-scale complex structures will be damaged during long service period, especially when exposed to harsh environment or natural calamities, such as winds and earthquakes. In cases of severe damage, these factors can lead to major accident and disastrous threat to human life safety [1]. Structural health monitoring (SHM) is concerned with performance monitoring of structures using various sensors and devices. SHM is also intended to evaluate the degradation rate and predict the remaining service life of a structure. An appropriate SHM system can help reduce the chance of catastrophic failure, maintenance cost, and down time for rehabilitation [2].

A typical SHM system includes three major components: a sensor system, a data processing system (including the data acquisition, transmission, and storage), and a health evaluation system [3]. The health status of structures can be surveilled, evaluated, and assessed by the overall system. Sensor system plays a key part in the SHM system. Owing to the cost limitation, it is impossible to place sensors in all candidate positions. Deploying fewer sensors on the structures and acquiring more health information is the focus of the study. How to place sensors reasonably has become one of the most importantly problems, which is known as optimal sensor placement (OSP). Kammer developed the effective independence (EFI) method which played close attention to the contribution of each sensor position to the linear independence of the identified modes [4]. Lim employed the method based on a given rank for the system observability matrix that satisfies modal test constraints to determine sensor positions [5]. Carne and Dohmann [6] proposed to use modal assurance criterion (MAC) matrix as a measure of the utility of a sensor configuration. Heo and Wang presented the modal kinetic energy (MKE) method and optimized the transducer placement of a long span bridge for identification and control purposes [7]. Miller introduced an approach to compute a Gaussian quadrature formula to give the optimal positions for sensors, and to illustrate the approach by a slewing beam [8]. Park et al utilized modal controllability and observability defined in balanced coordinate system to select the positions of sensors and actuators [9]. Meo and 
Zumpano modified the EFI method, and posed the effective independence driving-point residue (EFI-DPR) method for OSP to identify the vibration characteristics of the bridge [10]. Li et al combined the EFI method and MKE method, raised a quick EFI method through QR decomposition, and demonstrated the connections between EFI and MKE on the I-40 Bridge [11]. The meta-heuristic algorithm based on the swarm intelligence is especially appropriate and effective to solve the OSP problem. For example, genetic algorithms, monkey algorithm, particle swarm optimization, artificial bee colony algorithm, and ant colony optimization algorithm have been successfully applied in the OSP problem [12-20].

Artificial Fish Swarm Algorithm (AFSA) is a new kind of swarm intelligence optimization algorithm based on the behavior of fish [21-22]. The basic idea of AFSA is to imitate the fish behaviors such as prey, swarm and follow. Because AFSA is simple in principle and has some good features such as robustness, and tolerance of parameter setting, AFSA has been successfully applied to many kinds of optimization problems such as structural damage detection [23], multiple sequence alignment [24], network intrusion detection [25], optimization of drag and torque [26], optimization operation of cascade reservoirs [27], and symbolic regression [28].

In this paper, AFSA is adopted to cope with the OSP problem. The outline of the paper is as follows: Section 2 gives a brief description of AFSA. Section 3 establishes the finite element model (FEM) of an arch bridge structure and performs model analysis. Section 4 presents the OSP implementation in the above mentioned an arch bridge structure by using AFSA. Finally, a few concluding remarks are given.

\section{Description of AFSA}

\subsection{Principle of AFSA}

In water, fish are most likely distributed around the field with the most abundant food. A fish swarm completes its food foraging process by several social behaviors. AFSA, inspired by the behaviors of fish, is a novel method for searching the global optimum. It is a random search algorithm based on simulating fish swarm behaviors, which include prey behavior, swarm behavior, follow behavior and random behavior.

Suppose the current state of an artificial fish is $X=\left(x_{1}, x_{2}, \cdots, x_{n}\right)$, where $x_{i}(i=1,2, \cdots, n)$ is the variable to be optimized. $Y=f(X)$, the objective function, is the food concentration corresponding to $X . d_{i, j}=\left\|X_{i}-X_{j}\right\|$ is the distance between individual artificial fish $X_{i}$ and $X_{j}$. Visual is the perception scope of an artificial fish, which determines the moving direction of each artificial fish. When Visual becomes larger, the perception scope becomes more comprehensive. $\delta$ is the factor of crowding degree, which avoids more artificial fish getting together. Step is the maximum moving step length. To avoid missing the optimal solutions, Step should not be set too large. Try_number is the number of the biggest trial in prey behavior. $X_{v}=\left(x_{1}, x_{2}, \cdots, x_{n}\right)$ is the position at some moment. In the minimum problem, If $Y_{v}<Y$, the fish moves toward $X_{v}$ and arrives at the next position $X_{n e x t}$ according to equation (1). Otherwise, the fish continues to search other position within Visual. Visual and Step are shown in Figure 1.

$$
X_{\mathrm{next}}=X+\operatorname{rand}() \times \text { Step } \times \frac{X_{v}-X}{\left\|X_{v}-X\right\|}
$$

where $\operatorname{rand}()$ produces random number between 0 and 1. 


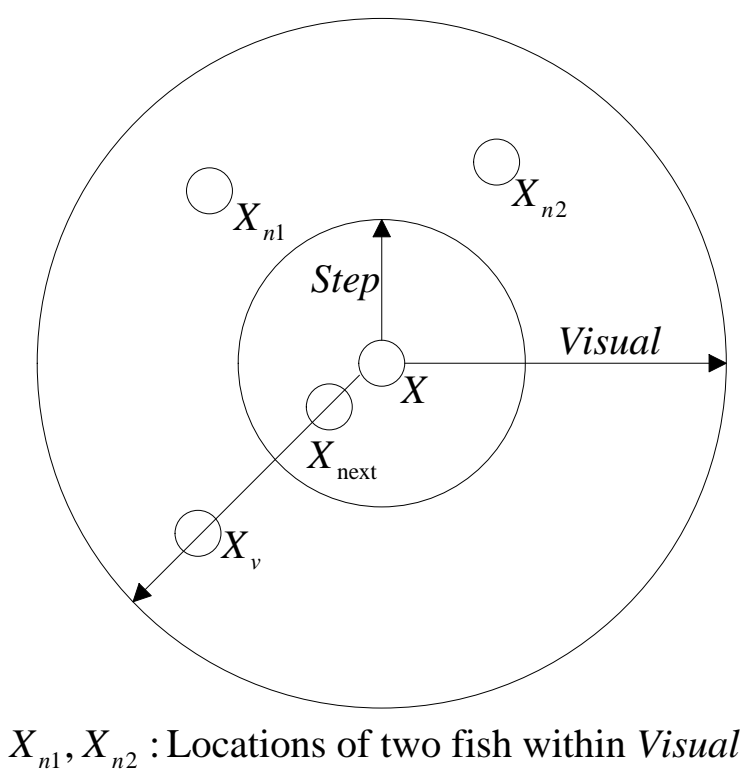

Figure 1. Visual and Step of Artificial Fish

\subsection{Flow chart of AFSA}

2.2.1. Prey behavior: An artificial fish follows the direction with high food concentration in prey behavior. Suppose the current state of an artificial fish is $X_{i}$ and its concentration is $Y_{i}$. Randomly select a new state $X_{j}$ within Visual according to equation (2).

$$
X_{j}=X_{i}+\operatorname{Visual} \times \operatorname{rand}()
$$

If $Y_{j}<Y_{i}$, the artificial fish moves toward $X_{j}$ with one Step according to equation (3)

$$
X_{i \mid \mathrm{next}}=X_{i}+\operatorname{rand}() \times \operatorname{Step} \times \frac{X_{j}-X_{i}}{\left\|X_{j}-X_{i}\right\|}
$$

If $Y_{j} \geq Y_{i}$, randomly reselect $X_{j}$ and judge whether it satisfies the condition of moving forward. If the condition cannot be satisfied after Try _number, the artificial fish randomly moves one step according to equation (4).

$$
X_{i \mid \mathrm{next}}=X_{i}+\text { Visual } \times \text { rand }()
$$

The prey behavior is shown in Figure 2.

2.2.2. Swarm behavior: An artificial fish tends to move toward the central position of fish swarm. This is the swarm behavior. Suppose the current state of an artificial fish is $X_{i}$; the total fish number is $n$. Search the current field $\left(d_{i, j}<\right.$ Visual $)$ to determine the partner number $n_{f}$ and central position $X_{c} . X_{c}$ is computed according to equation (5). Central food concentration is $Y_{c}$. 


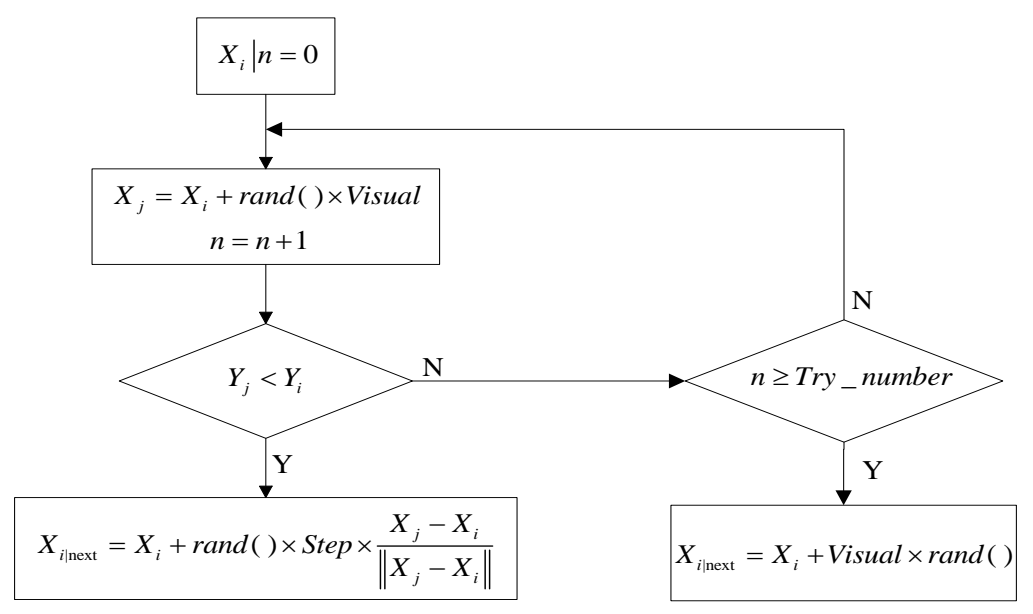

Figure 2. Prey Behavior

$$
X_{\mathrm{c}}=\frac{\sum_{j=1}^{n_{f}} X_{i}}{n_{f}}
$$

If $Y_{c}<Y_{i}$ and $\frac{n_{f}}{n}<\delta$, this indicates that there is more food and it is not too crowded at the center of the partners. So the artificial fish moves toward $X_{c}$ according to equation (6). Otherwise, the artificial fish performs prey behavior. The swarm behavior is illustrated in Figure 3.

$$
X_{i \mid \mathrm{next}}=X_{i}+\operatorname{rand}() \times \operatorname{Step} \times \frac{X_{c}-X_{i}}{\left\|X_{c}-X_{i}\right\|}
$$

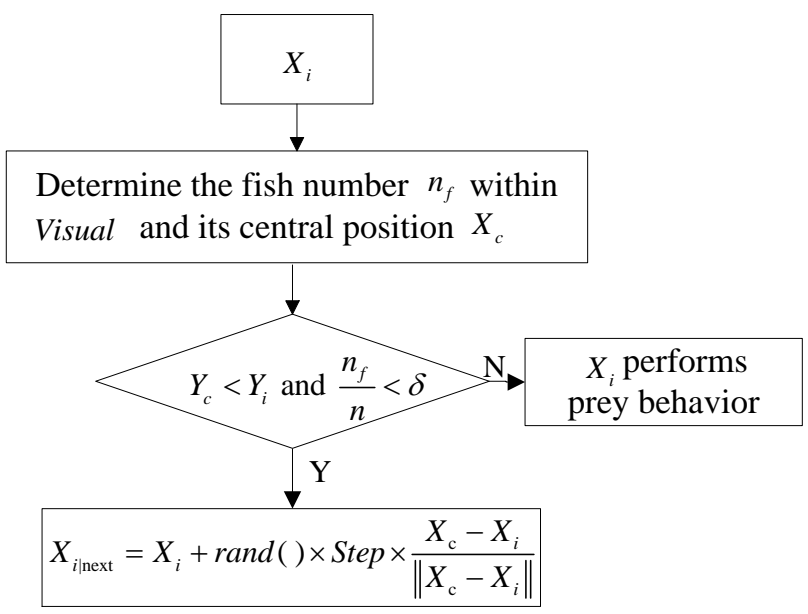

Figure 3. Swarm Behavior

2.2.3. Follow Behavior: When one or several fish find a field where there is more food and it is not crowded, the fish nearby will follow and move toward the food. This is the follow behavior. Suppose the current state of an artificial fish is $X_{i}$; the total number of fish is $n$. Search the current field $\left(d_{i, j}<\right.$ Visual $)$ to determine the partner $X_{j}\left(X_{\max }\right)$, which has the maximum $Y_{j}\left(Y_{\max }\right)$, from all partners. 
If $Y_{j}<Y_{i}$ and $\frac{n_{f}}{n}<\delta$, this indicates that there is more food and it is not too crowded at the position of partner $X_{\max }$. So the artificial fish moves toward partner $X_{\max }$ according to equation (7). Otherwise, the artificial fish performs prey behavior. The follow behavior is shown in Figure 4.

$$
X_{i \mid \text { next }}=X_{i}+\operatorname{rand}() \times \text { Step } \times \frac{X_{\text {max }}-X_{i}}{\left\|X_{\max }-X_{i}\right\|}
$$

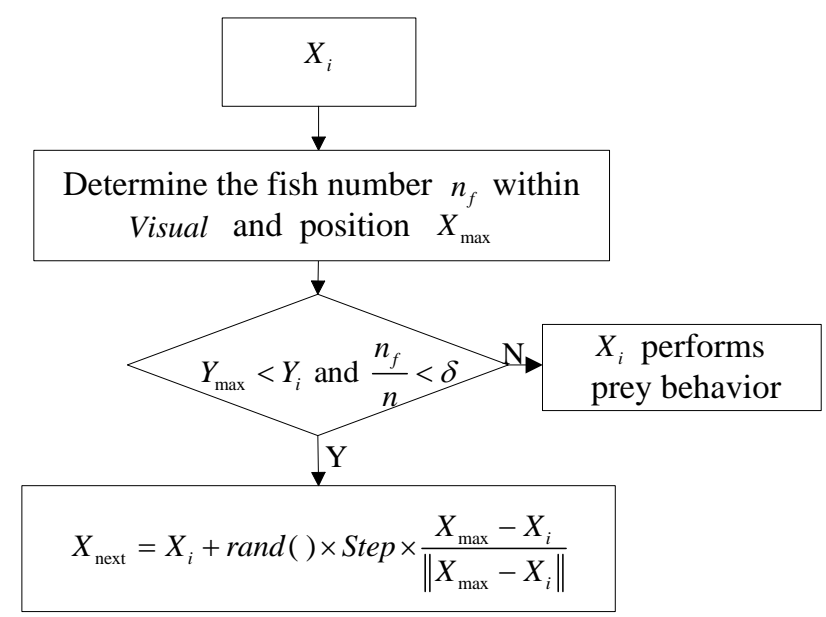

Figure 4. Follow Behavior

2.2.4. Random Behavior: This behavior is very simple. That is, randomly select a new state $X_{i \mid \text { next }}$ which is expressed as equation (8). And then the artificial fish moves toward that direction. Essentially, it is the default of prey behavior.

$$
X_{i \mid \mathrm{next}}=X_{i}+\operatorname{rand}() \times \text { Visual }
$$

\section{Modal Analysis on Arch Bridge}

As one kind of important complex structures, an arch bridge is selected to perform the OSP. Before applying AFSA to the OSP, modal analysis on the arch bridge is performed to gain the mode shapes.

\subsection{Description of Arch Bridge}

The diagram of an arch bridge is shown in Figure 5. The main span is $3350.98 \mathrm{~cm}$. Two side spans are both $2113.34 \mathrm{~cm}$. The main structure is composed of beams and plate materials. Elastic modulus of bridge floor is $\mathrm{E}=1.6 \times 10^{5} \mathrm{MPa}$. Poisson ration is 0.3 . Elastic modulus of materials for other beams is $\mathrm{E}=2.06 \times 10^{5} \mathrm{MPa}$.

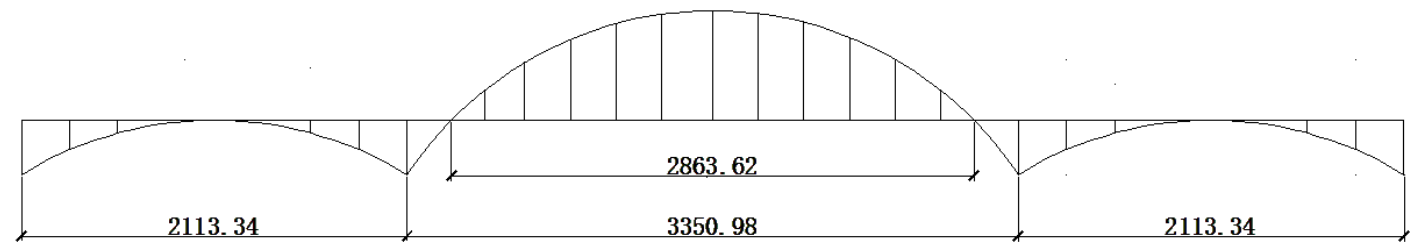

Figure 5. Diagram of Bridge Structure (unit: $\mathrm{cm}$ ) 


\subsection{Finite Element Model}

To obtain input data for OSP problem, a Finite Element Model (FEM) of the arch bridge is built by software ANSYS. The truss and the deck are simulated using beam elements and shell elements in the ANSYS element library. The model has 12631 frame elements and 11332 node elements. The three-dimension FEM developed is shown in Figure 6.

Modal analysis is performed to gain the mode shapes. Considering the low order modes have larger coefficients, the first 6 mode shapes are extracted, which are shown in Figure 7. And the first 6 modal frequencies are calculated. The first 6 modal frequencies and mode shape characteristics are given in Table 1.

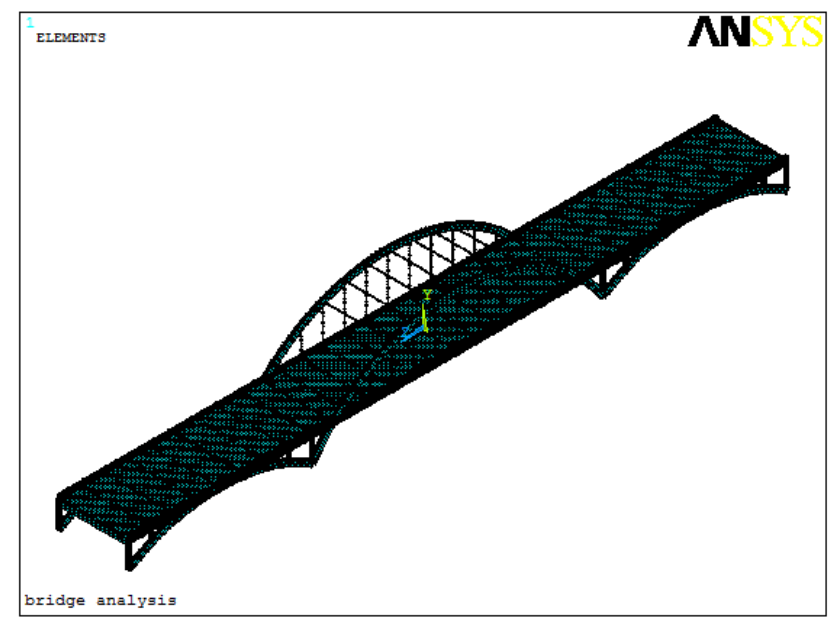

Figure 6. FEM of the Arch Bridge

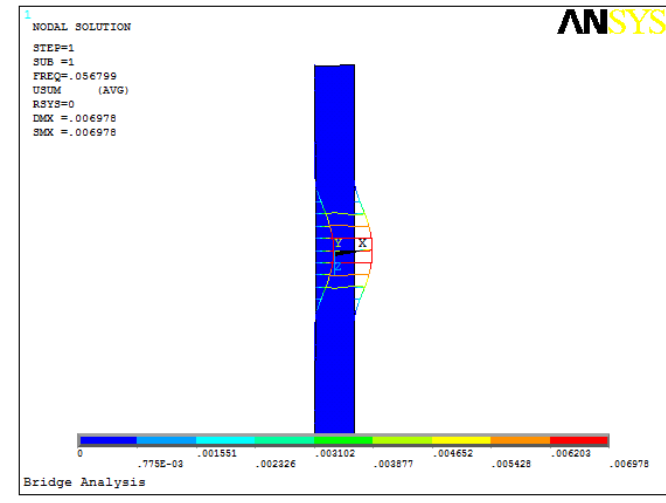

(a) The first mode shape

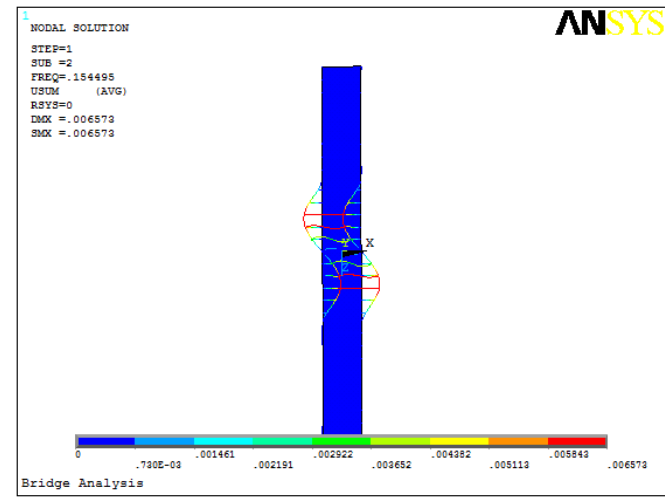

(b) The second mode shape 


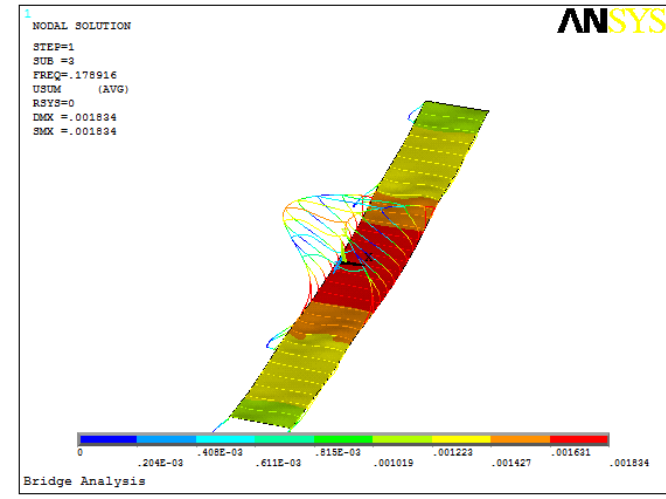

(c) The third mode shape

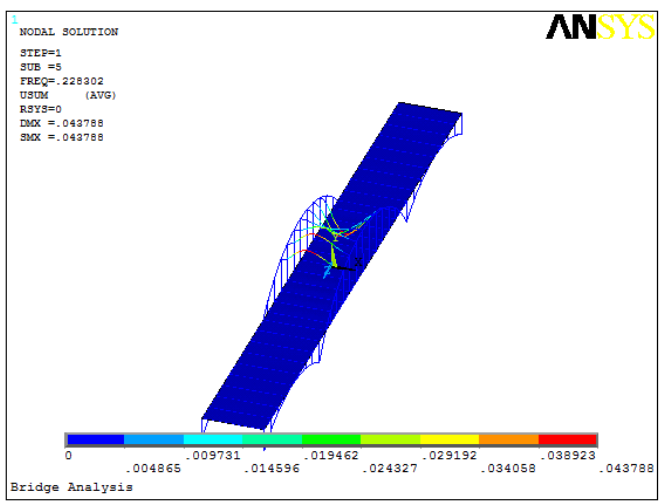

(e) The fifth mode shape

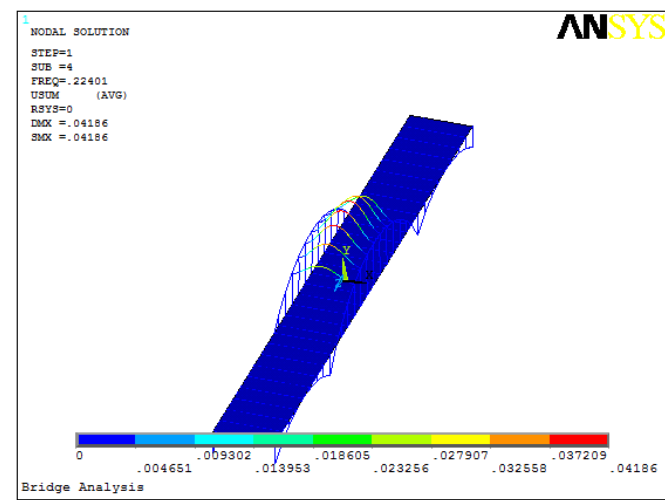

(d) The fourth mode shape

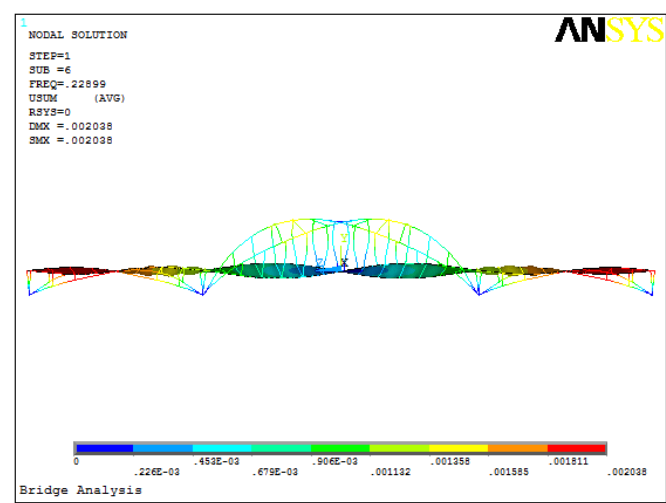

(f) The sixth mode shape

Figure 7. The First 6 Mode Shapes of the Arch Bridge

Table 1. The First 6 Modal Frequencies and Mode Shape Characteristic

\begin{tabular}{ccc}
\hline Order number & Frequency $/ \mathrm{Hz}$ & mode shape characteristic \\
\hline 1 & 0.05679 & Symmetric lateral bending of main arch \\
2 & 0.15450 & Anti-symmetric lateral bending of main arch \\
3 & 0.17892 & Symmetric vertical bending of main arch and deck \\
4 & 0.22401 & Symmetric vertical bending of main arch beam \\
5 & 0.22830 & Anti-symmetric vertical bending of main arch beam \\
6 & 0.22899 & Symmetric torsion of main arch and deck \\
\hline
\end{tabular}

\section{OSP for the Arch Bridge}

\subsection{Mathematical Model}

Suppose $\boldsymbol{\Phi}_{n \times l}$ is defined as the modal matrix which consists of the data from modal analysis, where $n$ is the number of degrees of freedom (DOFs), namely, DOFs of candidate sensor positions; $l$ denotes the order number of mode shapes. We need select $m$ DOFs from $n$ DOFs as the final sensor positions. In order to ensure the measured modal vectors being easily distinguished, modal assurance criterion (MAC) is applied to evaluate the measured modal vectors. The maximum off-diagonal elements of $\boldsymbol{M A C}$ as the objective function of minimum problem. The mathematical model can be expressed as

$$
\min f(\boldsymbol{X})=\max _{i \neq j}\left\{\boldsymbol{M A C}_{i j}\right\}
$$


where $0 \leq f(\boldsymbol{X}) \leq 1, \quad \boldsymbol{X}$ denotes an artificial fish; $\boldsymbol{M} \boldsymbol{A} \boldsymbol{C}_{i j}=\frac{\left(\boldsymbol{\phi}_{i}^{T} \boldsymbol{\phi}_{j}\right)^{2}}{\left(\boldsymbol{\phi}_{i}^{T} \boldsymbol{\phi}_{i}\right)\left(\boldsymbol{\phi}_{j}^{T} \boldsymbol{\phi}_{j}\right)}, \quad \boldsymbol{\phi}_{i}$ and $\phi_{j}$ represent the $i$ th and $j$ th column vectors in matrix $\boldsymbol{\Phi}$, respectively; and the superscript $T$ denotes the transpose of the vector. In equation (9), if the off-diagonal elements $\boldsymbol{M A C}_{i j}(i \neq j)$ tend to zero, it indicates that there is little correlation between the modal vector $i$ and the modal vector $j$, that is to say, the modal vector can be distinguished easily.

\subsection{Procedure of OSP}

The flow chart of AFSA for OSP is depicted in Figure 8.

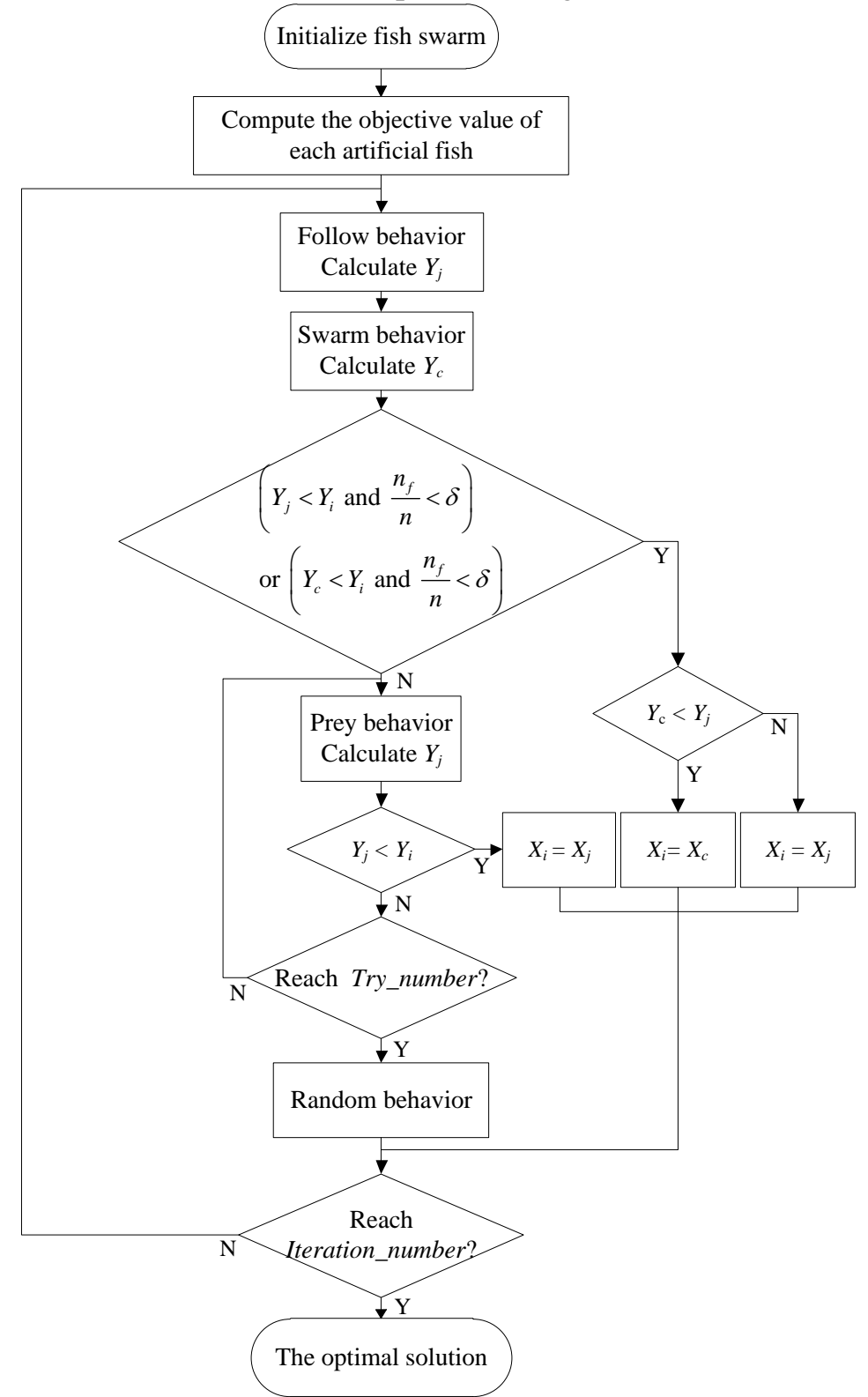

Figure 8. Flow Chart of AFSA for OSP 
(1) Initiate fish swarm. Randomly generate $n \times N$ initial fish swarm, where $n$ is the number of DOFs of the candidates of sensor nodes; $N$ is the size of artificial fish swarm. The value of each fish is a random number between 0 and 1 . Set number of maximum iterations, try number of prey behavior, perception scope, step, and factor of crowding degree as Iteration_number, Try_number, Visual, Step, and $\delta$ respectively.

(2) Get maximum off-diagonal element of $M A C$. On the basis of modal matrix, compute the value of objective function of each fish according to $\boldsymbol{\Phi}_{n \times l}$. Select $m(2<$ $m<n$ ) row and get maximum off-diagonal elements of $\boldsymbol{M A C}$, where $m$ is the final number of DOFs.

(3) Perform swarm and follow behaviors. Move toward the direction of smaller value of objective function. If the conditions of above two behaviors can not be satisfied, perform prey behavior. If Try_number is reached and the condition of moving forward still can not be satisfied, perform random behavior.

(4) Re-compute the values of objective function of each fish. If Iteration_number is reached, go to (5). Otherwise, go to (3).

(5) Determine the optimal solution according to equation (9).

\subsection{OSP Implementation}

Considering the symmetry of the arch bridge, only a quarter of bridge structure is selected to perform OSP. Remove the constraint nodes 10017, 10107 and take the rest 26 nodes. Each node has 3 translational DOFs which are represented respectively by $\mathrm{x}, \mathrm{y}, \mathrm{z}$. There are total $78(26 \times 3)$ translational DOFs. Adopt the first 6 mode shapes extracted in Section 3. The modal matrix is eventually constructed as $\boldsymbol{\Phi}_{78 \times 6}$. The candidates of sensor nodes are shown in Figure 9.

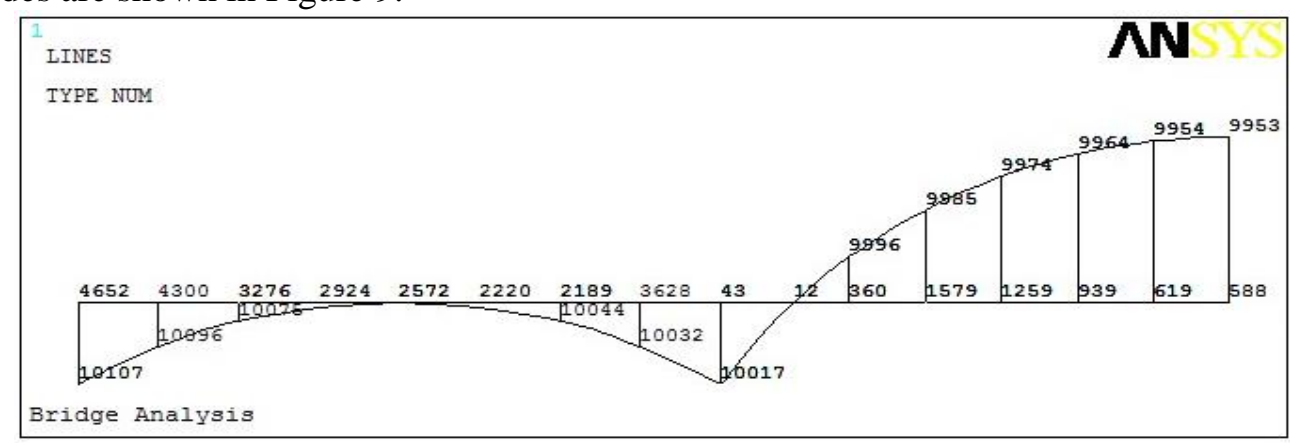

Figure 9. Candidates of Sensor Nodes

Parameters selection is of vital importance to the performance of AFSA. After trial and error, a group of optimal AFSA parameters are determined. These parameters are listed in Table 2. The maximum off-diagonal element of $\boldsymbol{M A C}$ matrix corresponding to each DOF is shown in Figure 10.

Table 2. Final AFSA Parameters for OSP

\begin{tabular}{cc}
\hline Parameter & value \\
\hline Size of artificial fish swarm $N$ & 100 \\
Try number of prey behavior Try_number & 15 \\
Number of maximum iterations Iteration_number & 150 \\
Perception scope Visual & 3 \\
Step Step & 1 \\
Factor of crowding degree $\delta$ & 1.5 \\
\hline
\end{tabular}


From Figure 10, when the DOF is between 2 and 10, the maximum value of the off-diagonal elements in the $\boldsymbol{M A C}$ matrix decreases rapidly. When the DOF is between 10 and 20 , the maximum value keeps stable. When the DOF is larger than 20 , the maximum value increases slowly. When the DOF is set to 10 , the maximum value of the off-diagonal elements reaches its minimum, and then the value increases slowly. Obviously, determine 10 as the numbers of sensors to be placed. The final sensor placement result of the arch bridge is given in Table 3.

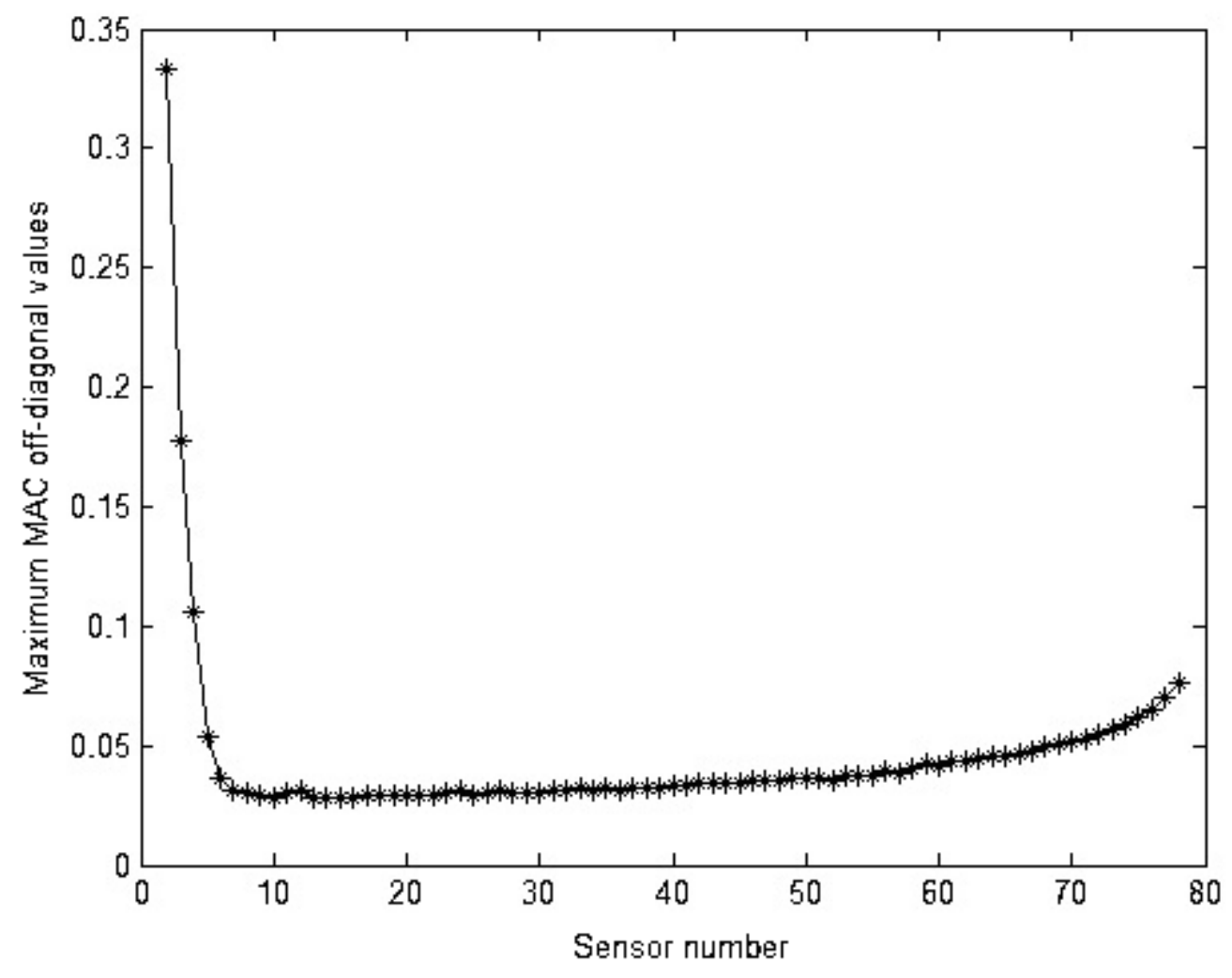

Figure 10. Curve of the Maximum Off-diagonal Element of MAC Matrix

Table 3. OSP for the Arch Bridge

\begin{tabular}{ccc}
\hline Sensor number & node & direction \\
\hline 1 & 9953 & $\mathrm{x}$ \\
2 & 939 & $\mathrm{x}$ \\
3 & 9953 & $\mathrm{y}$ \\
4 & 619 & $\mathrm{x}$ \\
5 & 9985 & $\mathrm{x}$ \\
6 & 3276 & $\mathrm{z}$ \\
7 & 10075 & $\mathrm{x}$ \\
8 & 588 & $\mathrm{y}$ \\
9 & 9996 & $\mathrm{y}$ \\
10 & 588 & $\mathrm{x}$ \\
\hline
\end{tabular}

\subsection{Comparison of the Methods}

To show the effectiveness of AFSA for OSP, AFSA and Particle Swarm Optimization (PSO) are carried out to compare their performances. The comparison of convergence curve between AFSA and PSO is shown in Figure 11. 
As shown in Figure 11, when iteration number is between 1and 7, AFSA rapidly converges to 0.0330 and then converges continuously. When the number of iterations reaches 48, but the maximum value of the off-diagonal elements in the MAC matrix by PSO is 0.0400 . Although the speed of AFSA is lower than that of PSO, AFSA is more adaptive than PSO, and the convergence precision of AFSA is higher than that of PSO. AFSA can effectively overcome the drawback of falling into local optimum. AFSA is more feasible to implement the OSP of the arch bridge.

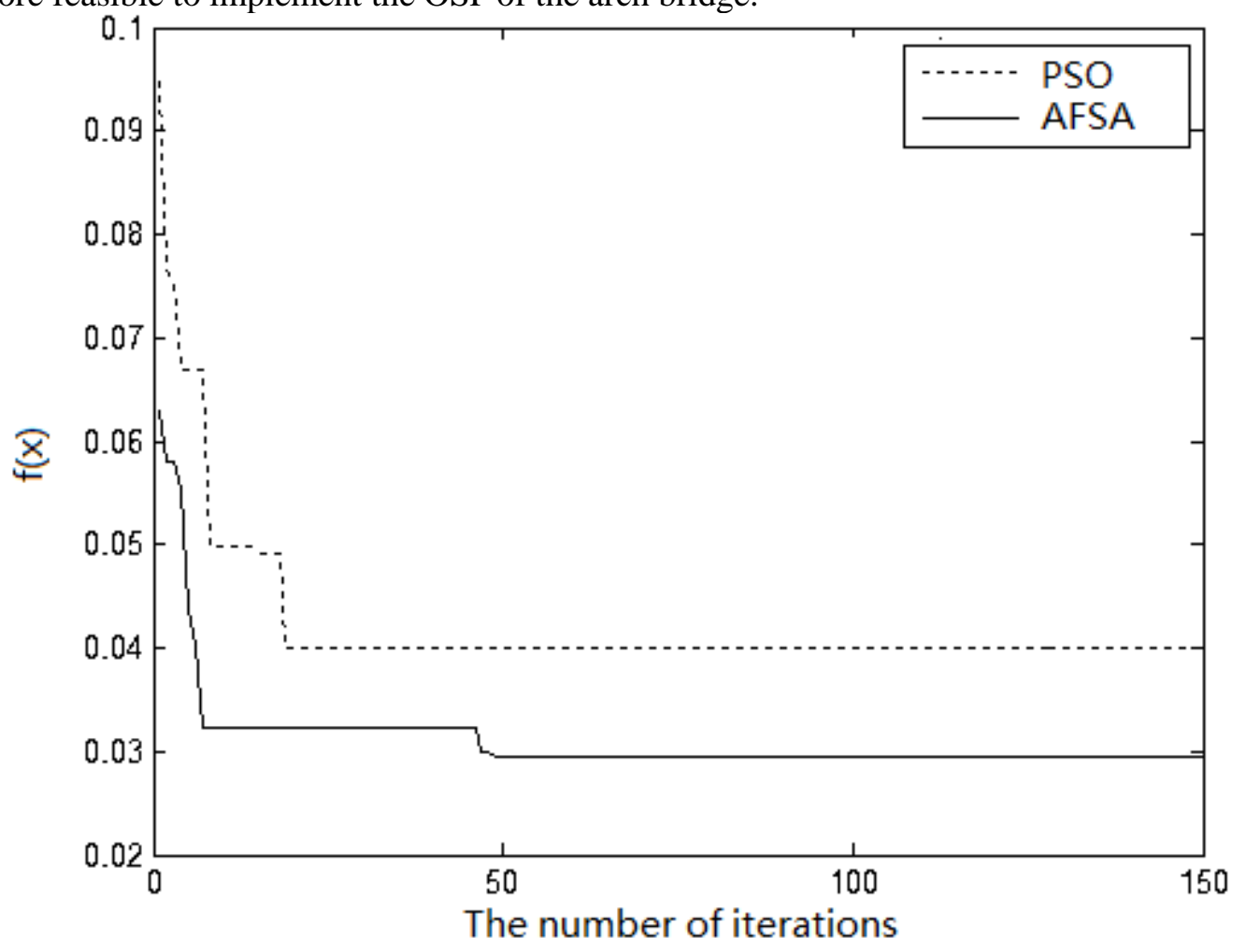

Figure 11. Comparison of Convergence Curve between AFSA and PSO

\section{Conclusions}

In this paper, AFSA is adapted to solve the OSP problem. Take an arch bridge to verify the proposed approach. Modal analysis on the arch bridge is carried out to extract the first 6 order mode shapes. The maximum off-diagonal elements of $\boldsymbol{M A C}$ as the objective function of minimum problem. Prey behavior, swarm behavior, follow behavior, and random behavior are means for searching the optimal solution. The AFSA parameters have direct influence on the sensor placement results. After trial and error, a group of optimal AFSA parameters are determined.

The result by AFSA, compared with that by PSO, shows the advantage of AFSA in the OSP problem. Some advantages are listed as follows: (1) Prey behavior: The search mechanism with in visual distance make the local search ability more powerful. (2) Swarm behavior and follow behavior: These mechanisms not only increase the probability of getting a highly-fit fish, but also keep the concentration of food at a certain level. (3) Random behavior: The mechanism makes the global search ability more powerful. 


\section{Acknowledgements}

This research is supported by National Natural Science Foundation of China (No. 61463028), Fundamental Research Funds for Colleges and Universities in Gansu Province (No. 213054), and Research Project of Gansu Education Department (No. 213027).

\section{References}

[1] B. B. Li, D. S. Li and X. F. Zhao, China Technological Sciences, vol. 12, no. 55, (2012).

[2] A. S. Noman, F. Deeba and A. Bagchi, Journal of Performance of Constructed Facilities, vol. 27, (2013).

[3] G. W. Housner, L. A. Bergman and T. K. Caughey, Journal of Engineering Mechanics, vol. 9, no. 123, (1997).

[4] D. C. Kammer, Journal of Guidance, Control and Dynamics, vol. 2, no. 14 (1991).

[5] K. B. Kim, Journal of Guidance, Control and Dynamics, vol. 1, no. 15, (1992).

[6] D. J. Demichele, Proceedings of the 13th International Modal Analysis Conference, (1995); Nashville, U.S.A.

[7] G. Heo, M. L.Wang and D. Satpathi, Soil Dynamics and Earthquake Engineering, vol. 7, no. 16, (1997).

[8] R. E. Miller, Applied Mathematics and Computation, vol. 1, no. 97, (1998).

[9] U. S. Park, J. W. Choi and W. S. Yoo, Journal of Mechanical Science and Technology, vol. 1, no. 17, (2003).

[10] M. Meo and G. Zumpano, Engineering Structures, vol. 10, no. 27, (2005).

[11] D. S. Li, H. N. Li and C. P. Fritzen, Journal of Sound and Vibration, vol. 4-5, no. 305, (2007).

[12] T. H. Yi, H. N. Li, M. Gu and X. D. Zhang, International Journal of Structural Stability and Dynamics, vol. 5 , no. 14, (2014).

[13] H. Y. Guo, L. Zhang and L. L. Zhang, Smart Materials and Structures, vol. 3, no. 13, (2004).

[14] W. Liu, W. C. Gao and Y. Sun, Journal of Sound and Vibration, vol. 1-2, p. 317, (2008).

[15] T. H. Yi, H. N. Li and M. Gu, Mathematical Problems in Engineering, (2011).

[16] L. C. Jiao and L. Wang, IEEE Transactions on System, Man and Cybernetics, Part A: System and Humans, vol. 5, no. 30, (2000).

[17] F. Kang, J. J. Liu and Q. Xu, Advanced Engineering Informatics, vol. 3, no. 22, (2008).

[18] Y. F. Liao, D. H. Yau and C. L.Chen, Computers \& Mathematics with Applications, vol. 5, no. 64, (2012).

[19] J. C. Bansal and K. Deep, Applied Mathematics and Computation, vol. 22, no. 218, (2012).

[20] J. Zhao, Q. L. Liu and W. Wang, Information Sciences, vol. 7, no. 181, (2011).

[21] X. L. Li, Z. J. Shao and J. X. Qian, "Systems Engineering-Theory and Practice, vol. 11, no. 22, (2002).

[22] Z. H. Huang and Y. D. Chen, International Journal of Control and Automation, vol. 5, no. 6, (2013).

[21] W. Wang, S. C. Gao and Z. Tang, Soft Computing: A Fusion of Foundations, Methodologies and Applications, vol. 12, no. 13, (2009).

[22] B. Naderi, M. Mousakhani and M. Khalili, International Journal of Advanced Manufacturing Technology, vol. 5-8, no. 66, (2013).

[23] L. Yu and C. Li, Advances in Structural Engineering, vol. 3, no. 17, (2014).

[24] W. H. Yang, Journal of Computational and Theoretical Nanoscience, vol. 3, no. 11, (2014).

[25] G. Wang and D. Dai, Journal of Computers, vol. 11, no. 8, (2013).

[26] T. F. Sun, D. L. Gao and Q. M. Liang, Journal of Mines, Metals and Fuels, vol. 11-12, no. 61, (2013).

[27] Y. Peng, Journal of Computers, vol. 4, no. 6, (2011).

[28] Q. Liu, T. Odaka, J. Kuroiwa and H. Ogura, IEICE Transactions on Information and Systems, vol. 4, (2013).

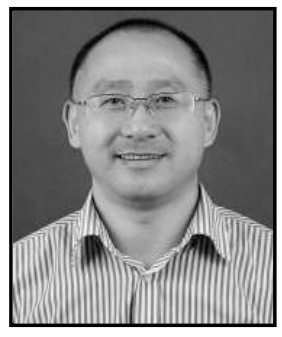

\section{Authors}

Zhen-rui PENG, he was born in Gansu Province, China, in 1972. He received the B.S. degree and M.S. degree from Lanzhou Jiaotong University, Lanzhou, in 1995 and in 2001 respectively, both in mechanical engineering. He received the Ph.D. degree from Zhejiang University, Hangzhou, in 2007, in control science and engineering. He is now with the school of mechatronic engineering, Lanzhou Jiaotong University. His research interests include measurement and control, intelligence optimization. 


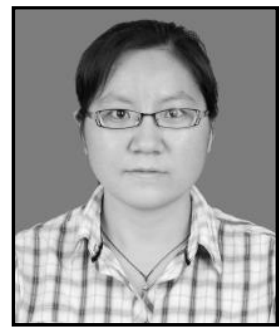

Yu ZHAO, she was born in Gansu Province, China, in 1990. She received the B.S. degree from Lanzhou Jiaotong University, Lanzhou, in 2011, in instrument science and engineering. She received the M.S. degree from Lanzhou Jiaotong University, Lanzhou, in 2014, in control science and engineering. Her research interests include detection technology and intelligence optimization.

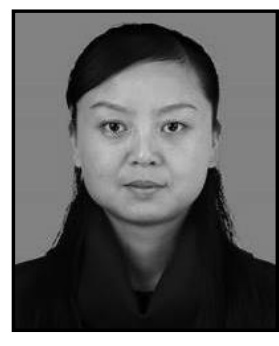

Hong YIN, she was born in Xinjiang Uygur Autonomous Region, China, in 1978. She received the B.S. degree from Lanzhou Jiaotong University, Lanzhou, in 2000, in mechanical engineering. She received the M.S. degree from Lanzhou Jiaotong University, Lanzhou, in 2003, in vehicle engineering. She is currently pursuing the $\mathrm{Ph} . \mathrm{D}$. degree with the school of mechatronic engineering, Lanzhou Jiaotong University. Her research interests include measurement and control, intelligence optimization.

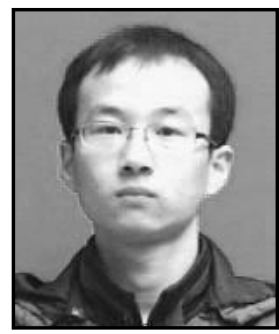

An PAN, he was born in 1986 and grew up in Gansu Province, China. He received the B.S. degree from North University of China, Taiyuan, in 2008, in optical information science and technology. He is currently pursuing the M.S. degree with the school of mechatronic engineering, Lanzhou Jiaotong University. He research interests include optimal sensor placement. 
International Journal of Control and Automation Vol. 8, No.4 (2015) 